Kovalenko Sergiy, Doctor of chemical sciences, professor, Department of Organic Chemistry, V. N. Karazin Kharkiv National University, 4 Svobody Sq., Kharkiv, Ukraine, 61022

E-mail: kovalenko.sergiy.m@gmail.com

Kovalenko Svitlana, Candidate of chemical sciences, associate professor, Department of Quality Management, National University of Pharmacy, 53 Pushkinska str., Kharkiv, Ukraine, 61002

E-mail: claire82@mail.ru

\author{
UDC 615.012:615.28]-092.4 \\ DOI: 10.15587/2313-8416.2016.65209
}

\title{
SYNTHESIS OF THE ROW OF NEW FUNCTIONAL DERIVATIVES OF 7-ARYLALKYL -8-HYDRAZINE THEOPHYLLINES
}

\section{(C) D. Korobko}

Hydrazine functional derivatives are widely used in medical practice as remedies applied for pharmacotherapy of depression, infection diseases, hypertension, diabetes, etc. It is worth mentioning that among obtained 7-R-8hydrazine derivatives of 1,3-dimethylxantine promising substances have been identified. Due to the fact that literature sources display only results of occasional studies of the reactions between 7-R-8-hydrazine theophyllines and mono- or dicarbonyle substances, the use of other keto reagents for xanthine bicycle at ${ }^{\text {th }}$ position functionalization will allow to explore synthetic potential of the last one, and with high probability may lead to obtaining original biologically active substances.

Aim. To study types of reaction between 8- hydrazinyl-1,3-dimethyl-7-aryl alkyl-1H-purine-2,6(3H,7H)-diones and a number of carbonyl containing reagents.

Methods. A nucleophilic addition reaction followed by dehydration or ethanol splitting was used, as well as the complex of the modern analysis methods to confirm the structure and individuality of the synthesized substances. Results. Different directions of 8-hydrazinyl-1,3,-dimethyl-7(fenetyl-, 3-phenylpropyl-, 3-phenylalyl)- 1H-purine$2,6(3 H, 7 H)$-diones chemical transformations in reactions with the appropriate carbonyl containing compounds have been studied experimentally. The structure of synthesized substances was confirmed by chromatography/mass and ${ }^{l} H$ NMR spectroscopy.

Conclusion. The group of 7-arylalkyl-8-(3,5-R, $R_{1}$-pyrazole-1-yl)theophyllines, consisting of two functionally substituted bioactive heterocycles, has been synthesized by reaction between initial substances and selected mono- and dicarbonyl compounds

Keywords: synthesis, 7,8-disubstituted of 1,3-dimethylxantine, hydrazine derivatives, spectral analysis methods

Функціональні похідні гідразину набули широкого застосування в медичній практиці як лікарські засоби для фармакокорекиії депресій, інфекиійних уражень, запальних процесів, гіпертензивних станів, иукрового діабету тощчо. Варто зазначити, щчо серед вже одержаних 7-R-8-гідразинопохідних 1,3диметилксантину також ідентифіковані перспективні у фармакологічному відношенні субстаниії. Оскільки з літератури відомо лише про спорадичні дослідження взаємодії 7-R-8-гідразинотеофілінів 3 моно- та дикарбонільними сполуками, використання інших кетовмісних реагентів з метою функціоналізації 8 положення ксантинового біциклу дозволить не тільки вивчити синтетичний потенціал останнього, але може з високою вирогідністю призвести до одержання нових біологічно активних речовин.

Мета. Дослідити напрямки взаємодії 8-гідразиніл-1,3-диметил-7-арилалкіл-1Н-пурин-2,6(3Н,7Н)-діонів 3 рядом карбонілвмісних реагентів.

Методи. Використано реакцію нуклеофільного приєднання з наступною дегідратацією чи відщепленням етанолу, а також комплекс сучасних спектральних методів аналізу для підтвердження структури й індивідуальності синтезованих речовин.

Результати. Експериментально встановлені окремі напрямки хімічних перетворень 8-гідразиніл-1,3диметил-7-(фенетил-, 3-фенілпропіл-, 3-фенілаліл)-1Н-пурин-2,6(3Н,7Н)-діонів в реакціях з відповідними карбонілвмісними сполуками. Структуру синтезованих речовин підтверджено даними хромато-масma ${ }^{1}$ Н ЯМР-спектрів.

Висновки. Ряд 7-арилалкіл-8-(3,5-R, $R_{1}$-піразол-1-іл)теофілінів, щзо складаються з двох функиіональнозаміщених біоактивних гетероциклів, синтезовані шляхом взаємодії вихідних речовин з окремими представниками моно- та дикарбонільних сполук

Ключові слова: синтез, 7,8-дизаміщені 1,3-диметилксантину, похідні гідразину, спектральні методи аналізу 


\section{Introduction}

Hydrazine functional derivatives are widely used in medical practice as remedies applied for pharmacotherapy of depression, infection diseases, hypertension, diabetes, etc [1-3]. Leading scientific journals constantly show new publications displaying results of both research in the area of hydrazine containing substances synthesis, and biological study for detection of original substances with promising pharmacological effects in the given group of compounds $[4,5]$. It is worth mentioning that theophylline molecule is also pharmacologically active. Its combination with different substituents, including heterocyclic number, may lead to increasing of both the severity and range of biological effects of the synthesized compounds. In this respect, a significant attention of scientists from different countries [6-8] is drawn to some 7-R-8-hydrazine derivatives of 1,3-dimethylxanthine, as basic structures for different chemical transformations.

2. Formulation of the problem in a general way, the relevance of the theme and its connection with important scientific and practical issues

Despite the large number of registered in Ukraine remedies, the need of original substances for treatment of different disorders of human's organism remains relevant. Therefore, the synthesis of new molecules (including different azaheterocycles derivatives) based on the use of already established «structure-activity» relationship is highly important for modern pharmaceutical and medical chemistry.

\section{Analysis of recent studies and publications in} which a solution of the problem and which draws on the author

Literature sources [2,3] describe a pronounced biological activity of the relevant xanthine and pyrazole methylated derivatives. Some representatives of these compounds have been successfully used in medical practice, but their synthetic and pharmacological potentials are far from being exhausted.

4. Allocation of unsolved parts of the general problem, which is dedicated to the article

Considering the theophylline derivatives' high reaction ability and versatile biological activity, the synthesis of the number of original 7,8-disubstituted of 1,3dimethylxantine was reasonable.

\section{Formulation of goals (tasks) of Article}

For this purpose, the reaction between investigated 8-hydrazinyl-1,3-dimethyl-7-arylalkyl-1H-purine-2,6(3H, $7 \mathrm{H}$ )-diones with a number of carbonyl containing reagents has been studied.

6. Statement of the basic material of the study (methods and objects) with the justification of the results

The initial 8-hydrazinyl-1,3,-dimethyl-7(fenetyl-, 3phenylpropyl-, 3-phenylalyl)- 1H-purine-2,6(3H,7H)-diones 5-7 have been produced with high output at hydrazinolysis of 7-arylalkyl-8-bromotheophyllines 2-4, which, in turn, have been synthesized by 8-bromotheophylline $\mathbf{1}$ alkylation by (2-bromoethyl)benzene, (3-chloropropyl)benzene, and (3-chlorprop-1-enyl)benzene in dimethylformamide medium in the presence of equimolecular proportion of sodium hydrogen carbonate [9].

The reaction of 5-7 compounds with methyl 4-(4methyl-(metoxy-,chloro-)phenyl)-2,4-dioxobutanoate and ethyl 3-(furan-2-yl)-3-oxopropanoate has been studied under different conditions of chemical process. The given reagents have not been chosen randomly, since they contain functional components, which adding to other heterocyclic systems led to strengthening of pharmacological activity of the last ones [10].

Literature sources $[11,12]$ show that 8 -hydrazine xanthine methylized derivatives react with acetyl- and benzoyl acetone at boiling in glacial acetic acid medium with formation of appropriate $8-\left(3,5-\mathrm{R}, \mathrm{R}_{1}\right.$-pyrazole-1yl)xantines.

However, in the case of the use of above mentioned esters as reagents, as results of analysis of the $R e$ axys Elsevier database relevant part show, reaction with 5-7 substances by the given method can be accompanied by the formation of a complex mixture of products.

It was found by experimental research, that the synthesis of methyl 3-(4-methylphenyl)-2-(2-(1,3-dimethyl-2,6dioxo-7-phenethyl-2,3,6,7-tetrahydro- $1 H$-purine-8-yl)hydrazono)propanoate 8 and methyl 3-(4-chlorophenyl)-2-(2(1,3-dimethyl-2,6-dioxo-7-(3-phenylalyl)-2,3,6,7-tetrahydro- $1 H$-purine-8-yl) hydrazono)propanoate 9 happens within 48 hours at room temperature in ethanol medium in the presence of catalytic amounts of hydrochloric acid (A, Fig. 1, Table 1) (it should be noted that reducing the reaction time leads to a reduction of target products output). Under the given conditions the formation of $\mathbf{B}$ and $\mathbf{C}$ structures is impossible, which is confirmed by chromatography/mass and ${ }^{1} \mathrm{H}$ NMR spectra (Fig. 1, Table 2). 
<smiles>[2H]Cn1c(NN=C([18OH])CC([18OH])=NNc2nc3c(c(=O)n(C)c(=O)n3C)n2C[2H])nc2c1c(=O)n(C)c(=O)n2C</smiles>

C<smiles>[2H]Cn1c(NN)nc2c1c(=O)n(C)c(=O)n2C</smiles><smiles></smiles><smiles>CC</smiles><smiles>Cn1c(=O)c2c(nc(N/N=C(/Br)CC(=O)Br)n2C)n(C)c1=O</smiles><smiles>CCn1c(-n2nc(Br)cc2C)nc2c1C(=O)C(=O)N2C</smiles><smiles></smiles>

$\mathrm{R}=\mathrm{CH}_{2}-\mathrm{C}_{6} \mathrm{H}_{5}, \mathrm{CH}_{2}-\mathrm{CH}_{2}-\mathrm{C}_{6} \mathrm{H}_{5}, \mathrm{CH}=\mathrm{CH}-\mathrm{C}_{6} \mathrm{H}_{5} ;$

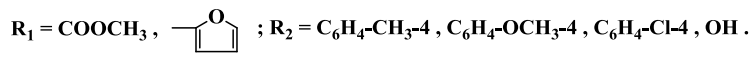

Fig. 1. Possible ways of reactions of the initial substances with mono- and dicarbonyle reagents

Table 1

Condensation product of

8-hydrazynil-7-(phenethyl, 3-phenylpropyl, 3-phenylalyl)-1,3-dimethyl-1H-purine-2,6(3H,7H)-dione Carbonyl mix of substances<smiles>[R]Cn1c(N/N=C(/CC([R2])=O)C(=O)OC)nc2c1c(=O)n(C)c(=O)n2C</smiles>

8,9<smiles>[R]Cn1c(-n2nc([R1])cc2[R2])nc2c1c(=O)n(C)c(=O)n2C</smiles>

10-21

\begin{tabular}{|c|c|c|c|c|c|c|}
\hline $\begin{array}{c}\text { No of } \\
\text { compounds }\end{array}$ & $\mathbf{R}$ & $\mathbf{R}_{1}$ & $\mathbf{R}_{2}$ & Yield, \% & T.pl., ${ }^{\circ} \mathbf{C}$ & Empirical Formula \\
\hline 8 & $\mathrm{CH}_{2}-\mathrm{C}_{6} \mathrm{H}_{5}$ & - & $\mathrm{C}_{6} \mathrm{H}_{4}-\mathrm{CH}_{3}-4$ & 94 & $175-177$ & $\mathrm{C}_{27} \mathrm{H}_{28} \mathrm{~N}_{6} \mathrm{O}_{5}$ \\
\hline 9 & $\mathrm{CH}=\mathrm{CH}-\mathrm{C}_{6} \mathrm{H}_{5}$ & - & $\mathrm{C}_{6} \mathrm{H}_{4}-\mathrm{Cl}-4$ & 91 & $189-191$ & $\mathrm{C}_{27} \mathrm{H}_{25} \mathrm{ClN}_{6} \mathrm{O}_{5}$ \\
\hline 10 & $\mathrm{CH}_{2}-\mathrm{C}_{6} \mathrm{H}_{5}$ & $\mathrm{COOCH}_{3}$ & $\mathrm{C}_{6} \mathrm{H}_{4}-\mathrm{CH}_{3}-4$ & 93 & 197-199 & $\mathrm{C}_{27} \mathrm{H}_{26} \mathrm{~N}_{6} \mathrm{O}_{4}$ \\
\hline 11 & $\mathrm{CH}_{2}-\mathrm{CH}_{2}-\mathrm{C}_{6} \mathrm{H}_{5}$ & $\mathrm{COOCH}_{3}$ & $\mathrm{C}_{6} \mathrm{H}_{4}-\mathrm{CH}_{3}-4$ & 95 & $168-170$ & $\mathrm{C}_{28} \mathrm{H}_{28} \mathrm{~N}_{6} \mathrm{O}_{4}$ \\
\hline 12 & $\mathrm{CH}=\mathrm{CH}-\mathrm{C}_{6} \mathrm{H}_{5}$ & $\mathrm{COOCH}_{3}$ & $\mathrm{C}_{6} \mathrm{H}_{4}-\mathrm{CH}_{3}-4$ & 91 & $157-159$ & $\mathrm{C}_{28} \mathrm{H}_{26} \mathrm{~N}_{6} \mathrm{O}_{4}$ \\
\hline 13 & $\mathrm{CH}_{2}-\mathrm{C}_{6} \mathrm{H}_{5}$ & $\mathrm{COOCH}_{3}$ & $\mathrm{C}_{6} \mathrm{H}_{4}-\mathrm{OCH}_{3}-4$ & 78 & 184-186 & $\mathrm{C}_{27} \mathrm{H}_{26} \mathrm{~N}_{6} \mathrm{O}_{5}$ \\
\hline 14 & $\mathrm{CH}_{2}-\mathrm{CH}_{2}-\mathrm{C}_{6} \mathrm{H}_{5}$ & $\mathrm{COOCH}_{3}$ & $\mathrm{C}_{6} \mathrm{H}_{4}-\mathrm{OCH}_{3}-4$ & 92 & $149-151$ & $\mathrm{C}_{28} \mathrm{H}_{28} \mathrm{~N}_{6} \mathrm{O}_{5}$ \\
\hline 15 & $\mathrm{CH}=\mathrm{CH}-\mathrm{C}_{6} \mathrm{H}_{5}$ & $\mathrm{COOCH}_{3}$ & $\mathrm{C}_{6} \mathrm{H}_{4}-\mathrm{OCH}_{3}-4$ & 87 & $95-97$ & $\mathrm{C}_{28} \mathrm{H}_{26} \mathrm{~N}_{6} \mathrm{O}_{5}$ \\
\hline 16 & $\mathrm{CH}_{2}-\mathrm{C}_{6} \mathrm{H}_{5}$ & $\mathrm{COOCH}_{3}$ & $\mathrm{C}_{6} \mathrm{H}_{4}-\mathrm{Cl}-4$ & 87 & $157-159$ & $\mathrm{C}_{26} \mathrm{H}_{23} \mathrm{ClN}_{6} \mathrm{O}_{4}$ \\
\hline 17 & $\mathrm{CH}_{2}-\mathrm{CH}_{2}-\mathrm{C}_{6} \mathrm{H}_{5}$ & $\mathrm{COOCH}_{3}$ & $\mathrm{C}_{6} \mathrm{H}_{4}-\mathrm{Cl}-4$ & 94 & $166-168$ & $\mathrm{C}_{27} \mathrm{H}_{25} \mathrm{ClN}_{6} \mathrm{O}_{4}$ \\
\hline 18 & $\mathrm{CH}=\mathrm{CH}-\mathrm{C}_{6} \mathrm{H}_{5}$ & $\mathrm{COOCH}_{3}$ & $\mathrm{C}_{6} \mathrm{H}_{4}-\mathrm{Cl}-4$ & 93 & 167-169 & $\mathrm{C}_{27} \mathrm{H}_{23} \mathrm{ClN}_{6} \mathrm{O}_{4}$ \\
\hline 19 & $\mathrm{CH}_{2}-\mathrm{C}_{6} \mathrm{H}_{5}$ & & $\mathrm{OH}$ & 86 & $172-174$ & $\mathrm{C}_{22} \mathrm{H}_{20} \mathrm{~N}_{6} \mathrm{O}_{4}$ \\
\hline 20 & $\mathrm{CH}_{2}-\mathrm{CH}_{2}-\mathrm{C}_{6} \mathrm{H}_{5}$ & & $\mathrm{OH}$ & 85 & $182-183,5$ & $\mathrm{C}_{23} \mathrm{H}_{22} \mathrm{~N}_{6} \mathrm{O}_{4}$ \\
\hline 21 & $\mathrm{CH}=\mathrm{CH}-\mathrm{C}_{6} \mathrm{H}_{5}$ & & $\mathrm{OH}$ & 84 & $191-193$ & $\mathrm{C}_{23} \mathrm{H}_{20} \mathrm{~N}_{6} \mathrm{O}_{4}$ \\
\hline
\end{tabular}


The results of ${ }^{1} \mathrm{H}$ NMR spectroscopic analysis of a number of original 7,8-disubstituted theophylline

Table 2<smiles>[R]Cn1c(NN=C([R1])CC([R4])=O)nc2c1c(=O)n(C)c(=O)n2C</smiles>

8,9<smiles>[R]Cn1c(-n2nc([R1])cc2[R1])nc2c1c(=O)n(C)c(=O)n2C</smiles>

10-21

\begin{tabular}{|c|c|c|c|c|c|c|}
\hline \multirow[b]{2}{*}{ № } & \multirow[b]{2}{*}{$\begin{array}{l}\text { Compo- } \\
\text { und }\end{array}$} & \multicolumn{5}{|c|}{ The values of the chemical shift, ppm } \\
\hline & & $\mathbf{N}^{7}-\mathbf{R}$ & $\begin{array}{c}\mathrm{N}^{1}-\mathrm{CH}_{3} \\
\mathrm{~N}^{3}-\mathrm{CH}_{3} \\
(3 \mathrm{H}, \mathrm{s})\end{array}$ & $\mathbf{R}_{\mathbf{1}}$ & $\mathbf{R}_{2}$ & Other protons \\
\hline 1 & 2 & 3 & 4 & 5 & 6 & 7 \\
\hline 1 & 8 & $\begin{array}{c}4,75 \mathrm{t}\left(2 \mathrm{H}, \mathrm{J}=7,5, \mathrm{~N}^{7}-\mathrm{C}_{\mathrm{H}_{2}}\right) \\
3,09 \mathrm{t}\left(2 \mathrm{H}, \mathrm{J}=6,7,-\mathrm{C} \underline{\mathrm{H}}_{2}-\mathrm{C}_{6} \mathrm{H}_{5}\right) \\
7,45-7,27 \mathrm{~m}(5 \mathrm{H}, \mathrm{C} \underline{\mathrm{H}} \text {-arom. }\end{array}$ & $\begin{array}{l}3,36 \\
3,23\end{array}$ & $\begin{array}{c}3,88 \mathrm{~s} \\
\left(3 \mathrm{H},-\mathrm{O}-\mathrm{C} \underline{H}_{3}\right)\end{array}$ & $\begin{array}{l}\text { 7,25-7,12m }(4 \mathrm{H} \\
\mathrm{C} \underline{H} \text {-arom.) } \\
2,26 \mathrm{~s}\left(3 \mathrm{H},-\underline{\mathrm{C}}_{3}\right)\end{array}$ & $\begin{array}{c}7,91 \mathrm{~s} \\
(1 \mathrm{H},-\mathrm{N} \underline{H}-) \\
2,41 \mathrm{~s}(2 \mathrm{H} \\
\left.=\mathrm{C}\left(\mathrm{R}_{1}\right)-\mathrm{C} \underline{H}_{2^{-}}\right) \\
\end{array}$ \\
\hline 2 & 9 & $\begin{array}{c}5,16 \mathrm{~d}\left(2 \mathrm{H}, \mathrm{J}=6,3, \mathrm{~N}^{7}-\mathrm{CH}_{2^{-}}\right) ; \\
6,41 \mathrm{~m}\left(1 \mathrm{H},-\mathrm{CH}_{2}-\mathrm{C} \underline{H}=\right) ; \\
\text { 6,61d (1H, J=15,8,-CH=CH}-) ; \\
7,38-7,29 \mathrm{~m}(5 \mathrm{H}, \mathrm{C} \underline{\mathrm{H}} \text {-arom. })\end{array}$ & $\begin{array}{l}3,33 \\
3,17\end{array}$ & $\begin{array}{c}3,86 \mathrm{~s} \\
\left(3 \mathrm{H},-\mathrm{O}-\mathrm{C} \underline{H}_{3}\right)\end{array}$ & $\begin{array}{c}\text { 7,65-7,57m (4H, } \\
\text { C } \underline{H} \text {-arom.) }\end{array}$ & $\begin{array}{c}8,04 \mathrm{~s} \\
(1 \mathrm{H},-\mathrm{N} \underline{H}-) \\
3,15 \mathrm{~s}(\underline{\mathrm{H}} \\
\left.=\mathrm{C}\left(\mathrm{R}_{1}\right)-\mathrm{C} \underline{H}_{2^{-}}\right) \\
\end{array}$ \\
\hline 3. & 10 & $\begin{array}{c}4,19 \mathrm{t}\left(2 \mathrm{H}, \mathrm{J}=7,2, \mathrm{~N}^{7}-\mathrm{CH}_{2^{-}}\right) ; \\
2,89 \mathrm{t}\left(2 \mathrm{H}, \mathrm{J}=7,0,-\mathrm{C} \underline{H}_{2}-\mathrm{C}_{6} \mathrm{H}_{5}\right) \\
7,21-7,13 \mathrm{~m}(5 \mathrm{H}, \mathrm{C} \underline{\mathrm{H}} \text {-arom. }\end{array}$ & $\begin{array}{c}3,32 \\
3,28\end{array}$ & $\begin{array}{c}3,92 \mathrm{~s} \\
\left(3 \mathrm{H},-\mathrm{O}-\mathrm{C} \underline{H}_{3}\right)\end{array}$ & $\begin{array}{c}7,23 \mathrm{~d}(2 \mathrm{H}, \mathrm{J}=7,9 \\
\mathrm{C}^{2} \underline{H}-, \mathrm{C}^{6} \underline{H} \text {-arom.); } \\
7,09 \mathrm{~d}(2 \mathrm{H}, \mathrm{J}=7,7 \\
\mathrm{C}^{3} \underline{H}-, \mathrm{C}^{5} \underline{H} \text {-arom.); } \\
2,29 \mathrm{~s} \\
\left(3 \mathrm{H},-\mathrm{C} \underline{H}_{3}\right)\end{array}$ & $\begin{array}{c}7,32 \mathrm{~s} \\
(1 \mathrm{H}, \mathrm{C} \underline{H} \text {-pyraz.) }\end{array}$ \\
\hline 4 & 11 & $\begin{array}{c}3,87 \mathrm{t}\left(2 \mathrm{H}, \mathrm{J}=7,7, \mathrm{~N}^{7}-\mathrm{C} \underline{H}_{2^{-}}\right) ; \\
1,76 \mathrm{t}\left(2 \mathrm{H}, \mathrm{J}=7,2,-\mathrm{C}_{H_{2}}-\mathrm{CH}_{2^{-}}\right) ; \\
2,42 \mathrm{t}\left(2 \mathrm{H}, \mathrm{J}=7,4,-\mathrm{CH}_{2}-\mathrm{C}_{2^{-}}\right) ; \\
7,20 \mathrm{~m}\left(2 \mathrm{H}, \mathrm{C}^{3} \underline{H}^{-}, \mathrm{C}^{5} \underline{H} \text {-arom.); }\right. \\
\text { 7,17d (2H, J=7,9, } \mathrm{C}^{2} \underline{H}-\mathrm{C}^{6} \underline{H} \text {-arom.); } \\
\text { 7,13d (1H, J=8,3, } \mathrm{C}^{4} \underline{H} \text {-arom.) }\end{array}$ & $\begin{array}{l}3,37 \\
3,25\end{array}$ & $\begin{array}{c}3,90 \mathrm{~s} \\
\left(3 \mathrm{H},-\mathrm{O}-\mathrm{C} \underline{H}_{3}\right)\end{array}$ & $\begin{array}{l}7,20 \mathrm{~m}\left(2 \mathrm{H}, \mathrm{C}^{2} \underline{H}-,\right. \\
\left.\mathrm{C}^{6} \underline{H} \text {-arom. }\right) ; 6,97 \mathrm{~d} \\
\left(2 \underline{\mathrm{H}}, \mathrm{J}=8,1, \mathrm{C}^{3} \underline{H}-\right. \\
\left.\mathrm{C}^{5} \underline{H} \text {-arom. }\right) ; 2,27 \mathrm{~s} \\
\left(3 \mathrm{H},-\mathrm{C} \underline{H}_{3}\right)\end{array}$ & $\begin{array}{c}7,27 \mathrm{~s} \\
\text { (1H, } \underline{\mathrm{H}}-\text {-pyraz.) }\end{array}$ \\
\hline 5 & 12 & $\begin{array}{c}\left.\text { 4,77d (2H, J=6,6, } \mathrm{N}^{7}-\mathrm{C} \underline{H}_{2^{-}}\right) ; \\
5,99 \mathrm{~m}\left(1 \mathrm{H},-\mathrm{CH}_{2}-\mathrm{C} \underline{H}=\right) ; \\
\left.\text { 6,26d (1H, J=16,2,-CH=C } \underline{H}_{-}\right) ; \\
7,25-7,20 \mathrm{~d}(3 \mathrm{H}, \mathrm{J}=7,7, \mathrm{C} \underline{H} \text {-arom.); } \\
7,16 \mathrm{~d}\left(2 \mathrm{H}, \mathrm{J}=7,8, \mathrm{C}^{3} \underline{H}-, \mathrm{C}^{5} \underline{H} \text {-arom.) }\right.\end{array}$ & $\begin{array}{l}3,39 \\
3,26\end{array}$ & $\begin{array}{c}3,86 \mathrm{~s} \\
\left(3 \mathrm{H},-\mathrm{O}-\mathrm{C} \underline{H}_{3}\right)\end{array}$ & $\begin{array}{c}7,27 \mathrm{~d}(2 \mathrm{H}, \mathrm{J}=7,6, \\
\mathrm{C}^{2} \underline{H}-, \mathrm{C}^{6} \underline{H} \text {-arom.); } \\
7,14 \mathrm{~d}(2 \mathrm{H}, \mathrm{J}=7,8 \\
\mathrm{C}^{3} \underline{H}-, \mathrm{C}^{5} \underline{H} \text {-arom.); } \\
2,24 \mathrm{~s} \\
\left(3 \mathrm{H},-\mathrm{C} \underline{H}_{3}\right)\end{array}$ & $\begin{array}{c}7,29 \mathrm{~s} \\
(1 \mathrm{H}, \mathrm{C} \underline{H} \text {-pyraz.) }\end{array}$ \\
\hline 6 & 13 & $\begin{array}{c}4,19 \mathrm{t}\left(2 \mathrm{H}, \mathrm{J}=7,4, \mathrm{~N}^{7}-\mathrm{C} \underline{H}_{2^{-}}\right) ; \\
2,89 \mathrm{t}\left(2 \mathrm{H}, \mathrm{J}=7,2,-\mathrm{C} \underline{H_{2}}-\mathrm{C}_{6} \mathrm{H}_{5}\right) ; \\
7,21-7,13 \mathrm{~m}(3 \mathrm{H}, \mathrm{C} \underline{H} \text {-arom.); } \\
7,08 \mathrm{~d}\left(2 \mathrm{H}, \mathrm{J}=7,7, \mathrm{C}^{3} \underline{H}-, \mathrm{C}^{5} \underline{H} \text {-arom.) }\right.\end{array}$ & $\begin{array}{l}3,33 \\
3,28\end{array}$ & $\begin{array}{c}3,91 \mathrm{~s} \\
\left(3 \mathrm{H},-\mathrm{O}-\mathrm{C} \underline{H}_{3}\right)\end{array}$ & $\begin{array}{c}7,23 \mathrm{~d}(2 \mathrm{H}, \mathrm{J}=8,3 \\
\mathrm{C}^{2} \underline{H}-, \mathrm{C}^{6} \underline{H} \text {-arom.); } \\
6,92 \mathrm{~d}(2 \mathrm{H}, \mathrm{J}=8,5 \\
\mathrm{C}^{3} \underline{H}-, \mathrm{C}^{5} \underline{H} \text {-arom.); } \\
3,74 \mathrm{~s}(3 \mathrm{H} \\
\left.\quad-\mathrm{O}-\mathrm{C} \underline{H}_{3}\right)\end{array}$ & $\begin{array}{c}7,28 \mathrm{~s} \\
(1 \mathrm{H}, \underline{\mathrm{C}} \underline{\text {-pyraz. }})\end{array}$ \\
\hline 7 & 14 & 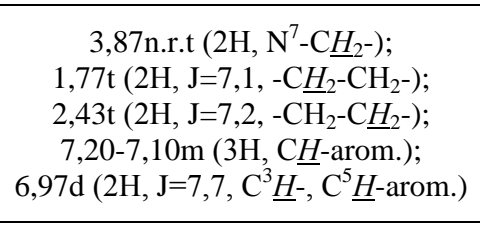 & $\begin{array}{l}3,38 \\
3,25\end{array}$ & $\begin{array}{c}3,90 \mathrm{~s} \\
\left(3 \mathrm{H},-\mathrm{O}-\mathrm{C} \underline{H}_{3}\right)\end{array}$ & $\begin{array}{c}7,23 \mathrm{~d}(2 \mathrm{H}, \mathrm{J}=8,1, \\
\mathrm{C}^{2} \underline{H}-, \mathrm{C}^{6} \mathrm{H} \text {-arom.); } \\
6,94 \mathrm{~d}(2 \mathrm{H}, \mathrm{J}=8,3 \\
\mathrm{C}^{3} \underline{H}-, \mathrm{C}^{5} \underline{H} \text {-arom.); } \\
3,74 \mathrm{~s}(3 \mathrm{H} \\
\left.-\mathrm{O}-\mathrm{C} \underline{H}_{3}\right)\end{array}$ & $\begin{array}{c}7,26 \mathrm{~s} \\
(1 \mathrm{H}, \mathrm{C} \underline{H} \text {-pyraz.) }\end{array}$ \\
\hline 8 & 15 & $\begin{array}{c}\text { 4,77d }\left(2 \mathrm{H}, \mathrm{J}=6,8, \mathrm{~N}^{7}-\mathrm{C}_{2_{2}}\right) \\
\text { 6,00m }\left(1 \mathrm{H},-\mathrm{CH}_{2}-\mathrm{C} \underline{H}=\right) ; \\
\text { 6,26d }(1 \mathrm{H}, \mathrm{J}=16,0,-\mathrm{CH}=\mathrm{C} \underline{H}-) ; \\
\text { 7,28-7,19m (3H, } \mathrm{C} \underline{H} \text {-arom. }) ; \\
\text { 7,15d }\left(2 \mathrm{H}, \mathrm{J}=8,1, \mathrm{C}^{3} \underline{H}-\mathrm{C}^{5} \underline{H} \text {-arom. }\right)\end{array}$ & $\begin{array}{l}3,39 \\
3,26\end{array}$ & $\begin{array}{c}3,85 \mathrm{~s} \\
\left(3 \mathrm{H},-\mathrm{O}-\mathrm{C} \underline{H}_{3}\right)\end{array}$ & $\begin{array}{c}\text { 7,28-7,19m }(2 \mathrm{H} \\
\mathrm{C}^{2} \underline{H}-, \mathrm{C}^{6} \mathrm{H} \text {-arom.); } \\
6,89 \mathrm{~d}(2 \mathrm{H}, \mathrm{J}=8,4 \\
\mathrm{C}^{3} \underline{H}-, \mathrm{C}^{5} \underline{H} \text {-arom.); } \\
3,70 \mathrm{~s}(3 \mathrm{H} \\
\left.-\mathrm{O}-\mathrm{C} \underline{H}_{3}\right)\end{array}$ & $\begin{array}{c}7,30 \mathrm{~s} \\
(1 \mathrm{H}, \mathrm{C} \underline{H} \text {-pyraz.) }\end{array}$ \\
\hline 9 & 16 & $\begin{array}{c}4,32 \mathrm{t}\left(2 \mathrm{H}, \mathrm{J}=7,2, \mathrm{~N}^{7}-\mathrm{C}_{\mathrm{H}_{2}}\right) \\
3,01 \mathrm{t}\left(2 \mathrm{H}, \mathrm{J}=7,0,-\mathrm{C} \underline{H}_{2}-\mathrm{C}_{6} \mathrm{H}_{5}\right) \\
7,30-7,09 \mathrm{~m}(5 \mathrm{H}, \mathrm{C} \underline{\mathrm{H}} \text {-arom. }\end{array}$ & $\begin{array}{l}3,33 \\
3,27\end{array}$ & $\begin{array}{c}3,93 \mathrm{~s} \\
\left(3 \mathrm{H},-\mathrm{O}-\mathrm{C} \underline{H}_{3}\right)\end{array}$ & $\begin{array}{l}7,42 \mathrm{~m}\left(2 \mathrm{H}, \mathrm{C}^{2} \underline{H}-\right. \\
\left.\mathrm{C}^{6} \underline{H} \text {-arom. }\right) ; 7,30- \\
7,09 \mathrm{~m}\left(2 \mathrm{H}, \mathrm{C}^{3} \underline{H}-\right. \\
\mathrm{C}^{5} \underline{H} \text {-arom. }\end{array}$ & $\begin{array}{c}7,42 \mathrm{~m} \\
\text { (1H, } \underline{\mathrm{C}} \underline{\text {-pyraz. }})\end{array}$ \\
\hline
\end{tabular}




\begin{tabular}{|c|c|c|c|c|c|c|}
\hline 1 & 2 & 3 & 4 & 5 & 6 & 7 \\
\hline 10 & 17 & 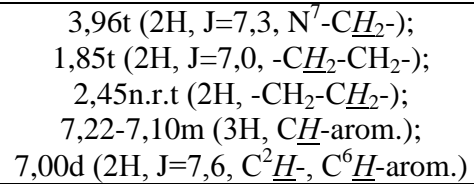 & $\begin{array}{l}3,34 \\
3,25\end{array}$ & $\begin{array}{c}3,91 \mathrm{~s} \\
\left(3 \mathrm{H},-\mathrm{O}-\mathrm{C} \underline{H}_{3}\right)\end{array}$ & $\begin{array}{c}7,47 \mathrm{~d}(2 \mathrm{H}, \mathrm{J}=7,9, \\
\left.\mathrm{C}^{2} \underline{H}-, \mathrm{C}^{6} \underline{H} \text {-arom. }\right) \\
7,35 \mathrm{~d}(2 \mathrm{H}, \mathrm{J}=8,1, \\
\mathrm{C}^{3} \underline{H}-, \mathrm{C}^{5} \underline{H} \text {-аром.) }\end{array}$ & $\begin{array}{c}7,38 \mathrm{~s} \\
(1 \mathrm{H}, \underline{\mathrm{C}} \underline{\text {-pyraz. }})\end{array}$ \\
\hline 11 & 18 & 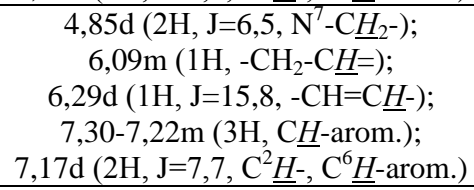 & $\begin{array}{l}3,36 \\
3,27\end{array}$ & $\begin{array}{c}3,87 \mathrm{~s} \\
\left(3 \mathrm{H},-\mathrm{O}-\mathrm{C} \underline{H}_{3}\right)\end{array}$ & $\begin{array}{l}7,38 \mathrm{~d}(2 \mathrm{H}, \mathrm{J}=8,1, \\
\mathrm{C}^{2} \underline{H}-, \mathrm{C}^{6} \underline{H} \text {-arom.); } \\
7,36 \mathrm{~d}(2 \mathrm{H}, \mathrm{J}=8,3, \\
\mathrm{C}^{3} \underline{H}-, \mathrm{C}^{5} \underline{H} \text {-arom.) }\end{array}$ & $\begin{array}{c}7,39 \mathrm{~s} \\
\text { (1H, } \underline{\mathrm{H}} \text {-pyraz.) }\end{array}$ \\
\hline 12 & 19 & $\begin{array}{c}4,38 \mathrm{t}\left(2 \mathrm{H}, \mathrm{J}=7,2, \mathrm{~N}^{7}-\mathrm{CH}_{2^{-}}\right) \\
3,05 \mathrm{t}\left(2 \mathrm{H}, \mathrm{J}=7,0,-\mathrm{CH}_{2}-\mathrm{C}_{6} \mathrm{H}_{5}\right) \\
7,28-7,12 \mathrm{~m}(5 \mathrm{H}, \mathrm{C} \underline{\mathrm{H}} \text {-arom. })\end{array}$ & $\begin{array}{l}3,45 \\
3,27\end{array}$ & $\begin{array}{l}\text { 7,80n.r.d (1H, } \\
\mathrm{C}^{5} \underline{H} \text {-fur.); } \\
6,89 \text { n.r.d (1H, } \\
\mathrm{C}^{4} \underline{H} \text {-fur.); } \\
6,64 \text { cn.r.d } \\
\text { (1H, } \mathrm{C}^{3} \underline{H} \text {-fur.) }\end{array}$ & $12,40 \mathrm{~s}(1 \mathrm{H},-\mathrm{O} \underline{H})$ & $\begin{array}{c}5,88 \mathrm{~s} \\
\text { (1H, } \underline{\mathrm{C}}-\text {-pyraz.) }\end{array}$ \\
\hline 13 & 20 & 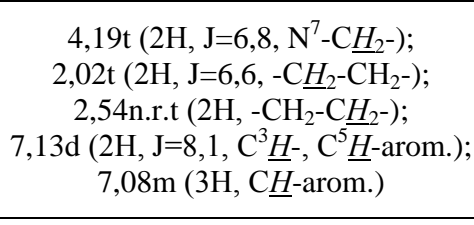 & $\begin{array}{l}3,42 \\
3,26\end{array}$ & $\begin{array}{l}\text { 7,76n.r.d (1H, } \\
\mathrm{C}^{5} \underline{H} \text {-fur.); } \\
6,84 \text { n.r.d (1H, } \\
\mathrm{C}^{4} \underline{H} \text {-fur.) } \\
\text { 6,61n.r.d (1H, } \\
\mathrm{C}^{3} \underline{H} \text {-fur.) } \\
\end{array}$ & $12,38 \mathrm{~s}(1 \mathrm{H},-\mathrm{O} \underline{H})$ & $\begin{array}{c}5,84 \mathrm{~s}(1 \mathrm{H}, \mathrm{C} \underline{H}- \\
\text { pyraz. })\end{array}$ \\
\hline 14 & 21 & $\begin{array}{c}\left.\text { 5,03d (2H, J=6,7, N } \mathrm{N}^{7}-\mathrm{CH}_{2^{-}}\right) \\
\text {6,32m (2H, }-\mathrm{C} \underline{H}=\mathrm{C} \underline{H}-) \\
7,25 \mathrm{~m}(5 \mathrm{H}, \mathrm{C} \underline{H} \text {-arom. })\end{array}$ & $\begin{array}{l}3,41 \\
3,24\end{array}$ & $\begin{array}{l}\text { 7,75n.r.d (1H, } \\
\mathrm{C}^{5} \underline{H} \text {-fur.); } \\
6,86 \text { n.r.d (1H, } \\
\mathrm{C}^{4} \underline{H} \text {-fur.); } \\
\text { 6,57n.r.d (1H, } \\
\mathrm{C}^{3} \underline{H} \text {-fur.) }\end{array}$ & $12,39 \mathrm{~s}(1 \mathrm{H},-\mathrm{O} \underline{H})$ & $\begin{array}{c}5,85 \mathrm{~s} \\
\text { (1H, } \underline{\mathrm{H}}-\text {-pyraz.) }\end{array}$ \\
\hline
\end{tabular}

Results of the study of reaction between 5-7 compounds and ethyl 3-(furane-2-yl)-3-oxopropanoate occurred to be interesting. Usually, heating of hydrazine derivatives with the given type of dicarbonile compounds for 1-3 hours in alcohol medium under acid catalysis conditions is realized by formation of the appropriate esters of hydrazono-3- $R$-propanoic acids $[10,13]$. Nevertheless, in all cases there were selected from the reaction mixtures that type of products, which were identified as 8-(3-furane-2-yl)-5-hydroxy-1H-pyrazole-1-yl)-1,3-dimethyl-7-(fenetyl-, 3-phenylpropyl-, 3-phenylalyl)- 1H-purine-2,6(3H,7H)-diones 19-21 (D, Fig. 1, Table 1). Chromatography/mass and ${ }^{1} \mathrm{H}$ NMR spectra completely correlate with the proposed structure $\mathbf{D}$ (Fig. 1, Table 2).

To implement the plan of synthetic studies, reaction between 5-7 substances and methyl 4-(4-methyl(metoxy-, chloro-)phenyl)-2,4-dioxobutanoate) has been studied. Theoretically, formation of five products (A-E, Fig. 1) is possible in the given reaction under various conditions (medium used, duration of transformation, temperature, etc.). It has been shown, that boiling for 8 hours of the mixture components in glacial acetic acid medium may lead to cyclocondensation and production of relevant pyrazole derivatives with high output 10-18 (Fig. 1, Tab. 1) of the $\mathbf{D}$ structure, which is confirmed by spectral analysis results (Tab. 2). The synthesized substances (8-21) are white $(\mathbf{8}, \mathbf{1 0 - 1 4}, \mathbf{1 6 - 1 8})$, greenishyellow (9), pale brown $(\mathbf{1 5}, \mathbf{1 9 - 2 1})$, insoluble in water, soluble in alcohols, dioxane, dimethylformamide.

Chromatography/mass spectroscopy research of 8-21 compounds under «soft» ionization conditions allowed to register appropriate peaks of quasi molecular ions $[\mathrm{MH}]^{+}$of high intensity.
IR-spectra of 11, 12, 14, 17, 18, 20 compounds are characterized by intensive absorption of ester group of pyrazolile residue at 1742,9-1730,8 $\mathrm{sm}^{-1}$ (except 20), the same absorption lines of xanthine fragment $\mathrm{CO}$ groups of molecules at 1708,7-1706,7 $\mathrm{sm}^{-1}$ and 1672,2$1651,9 \mathrm{sm}^{-1}$, respectively, stretching vibrations of aromatic $\mathrm{CH}$-connections as low intense lines 3137,6$3021,6 \mathrm{sm}^{-1}$. For 20 substance absorption lines at $3152,0 \mathrm{sm}^{-1}$ and 1555,9 $\mathrm{sm}^{-1}$ and intense deformation vibrations at $741,2 \mathrm{sm}^{-1}$ were registered, which is caused by the presence of furane cycle in its structure and completely correlated with literature [14].

The analysis of ${ }^{1} \mathrm{H}$ NMR spectra of the synthesized compounds 8-21 allowed proofing their structure clearly. For $\mathbf{8}$ and $\mathbf{9}$ substances one proton singlets at 8,04-7,91 ppm and 3,15-2,41 ppm were specific, due to the presence of - $\mathrm{NH}$ - and $-\mathrm{CH}_{2}$-groups for substituent in the $8^{\text {th }}$ position of molecules, respectively. In ${ }^{1} \mathrm{H}$ NMR spectra of 10-21 compounds pyrazole residue proton resonates as singlet at 7,42-5,84 ppm, and its chemical shift depends on the $R_{1}$ and $R_{2}$ radicals. Three one proton unsplit doublets, resonating at 7,80-7,75 ppm, 6,896,84 ppm and 6,64-6,57 ppm, respectively, were specific for substances 19-21, that allows to identify furane fragments of molecule [15].

In ${ }^{1} \mathrm{H}$ NMR spectra of compounds 8 and 9 double signals of protons of - $\mathrm{NH}-,-\mathrm{CH}_{2}-\mathrm{Ta}-\mathrm{OCH}_{3}$-groups were registered, indicating possible existence of these substances as mixtures of E- and Z-isomers. Due to $\mathrm{OH}$ group presence, in weak fields of ${ }^{1} \mathrm{H}$ NMR spectra of 1921 compounds expanded one proton singlets were identified, which confirmes the presence of the given compounds in enol form. 
Chromatography/mass spectroscopy research of the obtained substances were carried out using HPLC Agilent 1100 Series, equipped with diode matrix and mass-selective Agilent LC/MSD SL detectors. Chemical ionization at atmospheric pressure was chosen as ionisation method. The ${ }^{1} \mathrm{H}$ NMR spectra of synthesized compounds were recorded by using Varian Mercury 400 (400 MHz) spectrometer, DMSO- $\mathrm{d}_{6}$ solvent, internal standard - tetrametylsylan. IR spetra (in area of 4000-600 sm $\mathrm{sm}^{-1}$ ) were recorded by using Bruker ALPHA FT-IR spectrometer on the ATR console (soothing a general display).

8-hydrazynyl-7-phenethyl-(5), 3-phenylpropyl-(6), 3phenylalyl-(7))-1,3-dimethyl-1H-purine-2,6(3H,7H)-diones

Add $2.43 \mathrm{ml}$ hydrazine hydrate $(0,05$ mole at $\rho=$ $\left.=1,03 \mathrm{~g} / \mathrm{sm}^{3}\right)$ to $3.63 \mathrm{~g}$ (0.01 mole) substance 2 (to $3.77 \mathrm{~g}$ (0.01 mole) substance 3 or to $3.75 \mathrm{~g}$ ( $0.01 \mathrm{~mole})$ substance 4) in $18 \mathrm{ml}$ of the dioxane-water mixture $2: 1$. The obtained mixtures are heated for 4-5 hours. As soon as it cooled forcibly, yellowish or orange solutions are poured into water. Crystal precipitates are formed immediately; they are filtered and purified for the analysis by recrystallization from propanol-2 (5), and mixtures of ethanolwater 2:1 (6), ethanol-dioxane 5:1 (7).

Methyl 3-(4-methylphenyl-(8), 4-chlorophenyl-(9))2-(2-(1,3-dimethyl-2,6-dioxo-7-(phenethyl-(8), 3-phenylalyl(9))-2,3,6,7-tetrahydro-1H-purine-8-yl)hydrazono)propanoaty (Table 1)

The mixture of 0.002 mole of substance 5 or 7 , 0.002 mole of methyl 4-(4-methyl-(chlorophenyl)-2,4dioxobutanoate and 2 drops of hydrochloric acid in $15 \mathrm{ml}$ of ethanol is set aside at room temperature for 48 hours. Flasks content is diluted with water; formed precipitates are filtered, dried, and recrystallized from ethanol.

Methyl 1-(1,3-dimethyl-2,6-dioxo-7-arylalkyl-2,3,6,7tetrahydro-1H-purine-8-yl)-5-(4-R-phenyl)-1H-pirazole-33carboxylaty (10-18, Table 1).

Add 0.0027 mole of relevant methyl 2,4-dioxo-4R-phenylbutanoate $\left(\mathrm{R}=\mathrm{CH}_{3}, \mathrm{OCH}_{3}, \mathrm{Cl}\right)$ to suspension of 0.0027 mole of substances 5-7 in $7 \mathrm{ml}$ of glacial acetic acid and boil for 8 hours. Obtained solutions with intensive colors are cooled and poured into water. Formed precipitates are filtered, dried, and purified by recrystallization from ethanol.

8-(3-(Furane-2-yl)-5-hydroxy-1H-pyrazole-1-yl)-1, 3dithyl-7-(phenethyl-(19), 3-phenylpropyl-(20), 3-phenylalyl-(21))-1H-purine-2,6(3H,7H)-diones (Table 1).

Add $0,365 \mathrm{~g}$ (0.002 mole) (0.40 g (0.0022 mole) ethyl-3-(furane-2-yl)-oxopropanoate and 2 drops of hydrochloric acid to $0.63 \mathrm{~g}$ (0.002 mole) of substance 5 $(0,72 \mathrm{~g}(0.0022 \mathrm{~mole})$ of substances 6 or 7$)$ in $10-12 \mathrm{ml}$ of propanol. Obtained mixtures are heated for 2 hours. As soon as flasks content is cooled, it should be poured into water. Precipitates of 19-21 substances that formed, are filtered, and purified for the analysis by crystallization from ethanol.

\section{Conclusion}

Different directions of 8-hydrazinyl-1,3,-dimethyl-7(fenetyl-, 3-phenylpropyl, 3-phenylalyl)- 1H-purine-2,6(3H,7H)-diones chemical transformations in reac- tions with the appropriate carbonyl containing compounds have been studied experimentally. The structure of synthesized substances was confirmed by the complex of modern spectral analysis methods. The obtained 7-arylalkyl8-(3,5- $R, R_{l}$-pyrazole-1-yl)theophyllines, consisting of two functionally substituted bioactive heterocycles, are promising enough for the further biological research.

\section{References}

1. Zelenin, K. N. Gidrazin [Text] / K. N. Zelenin // Sorosovskij obrazovatel'nyj zhurnal. - 1998. - Vol. 5. - P. 59-65.

2. Mashkovskij, M. D. Lekarstvennye sredstva [Text] / M. D. Mashkovskij; 16th edition. - Moscow: Novaja volna, 2012. -1216 p.

3. Martindale: The Complete Drug Reference [Text] / S. C. Sweetman (Ed.); Thirty-sixth edition. - Pharmaceutical Press, 2009. -3694 p.

4. Rollas, S. Biological Activities of Hydrazone Derivatives [Text] / S. Rollas, S. Güniz Küçükgüzel // Molecules. 2007. - Vol. 12, Issue 8. - P. 1910-1939. doi: 10.3390/12081910

5. Narang, R. Review on Biological Activities and Chemical Synthesis of Hydrazide Derivatives [Text] / R. Narang, B. Narasimhan, S. A. Sharma // Current Medicinal Chemistry. - 2012. - Vol. 19, Issue 4. - P. 569-612. doi: 10.2174/092986712798918789

6. Mosselhi, M. A. N. New [e]-Fused Caffeines: A Simple Synthesis of 3-Substituted [1,2,4]Triazolo[4,3- e ]purines [Text] / M. A. N. Mosselhi, N. M. Tawfik, A. S. Shawali // Monatshefte fur Chemie/Chemical Monthly. - 2003. Vol. 134, Issue 4. - P. 565-571. doi: 10.1007/s00706-002-0514-7

7. Petch, D. Design and synthesis of EGFR dimerization inhibitors and evaluation of their potential in the treatment of psoriasis [Text] / D. Petch, R. J. Anderson, A. Cunningham, S. E. George, D. E. Hibbs, R. Liu et. al. // Bioorganic and Medicinal Chemistry. - 2012. - Vol. 20, Issue 19. - P. 5901-5914. doi: 10.1016/j.bmc.2012.07.048

8. Ashour, F. A. Synthesis, anticancer, anti-HIV-1, and antimicrobial activity of some tricyclic triazino and triazolo[4,3-e]purine derivatives [Text] / F. A. Ashour, S. M. Rida, S. A. M. El-Hawash, M. M. El-Semary, M. H. Badr // Medicinal Chemistry Research. - 2102. - Vol. 21, Issue 7. - P. 11071119. doi: 10.1007/s00044-011-9612-6

9. Korobko, D. B. Syntez i fizyko-himichni vlastyvosti dejakyh 7-aralkil-(alkenil)-8-bromo-(tio-)teofiliniv [Text]: materialy XXVIII vseukr. naukovo-prakt. konf. / D. B. Korobko, O. V. Berezovs'kyj, M. M. Palagnjuk, Je. S. Pylypiv // Liky ljudyni. - Harkiv, 2011. - P. 263-269.

10. Abdel-Wahab, B. F. Utility of 2,4-dioxoesters in the synthesis of new heterocycles [Text] / B. F. Abdel-Wahab, H. Abdel-Gawad, H. A. Mohamed, K. M. Dawood // HETEROCYCLES. - 2010. - Vol. 81, Issue 1. - P. 1-55. doi: 10.3987/ rev-09-659

11. Gumennoj, V. P. Kondensacija 1,3-dikarbonil'nyh soedinenij s 8-gidrazinoksantinami [Text] / V. P. Gumennoj // Chem. Abstrac. - 1984. - Vol. 101, Issue 13. - P. 110869.

12. Povstjanoj, M. V. Kondensirovannye imidazo1,2,4-aziny. XXVI. O reakcii 8-gidrazinoksantina i 8-(1metilgidrazino)teofillina s acetil- i benzoilacetonom [Text] / M. V. Povstjanoj, V. P. Kruglenko, N. A. Kljuev et. al. // Zhurnal org. himii. - 1992. - Vol. 28, Issue 4. - P. 849-856.

13. Voskobojnik, O. Ju. Syntez, peretvorennja, fizykohimichni ta biologichni vlastyvosti [\{2-R- $(3 \mathrm{H})-[$ hinazolin-4iliden \}gidrazono]karbonovyh kyslot [Text]: avtoref. dys. ... kand. farm. nauk : spec. / O. Ju. Voskobojnik. - L'viv. nac. med. un-t imeni Danyla Galyc'kogo, 2008. - 26 p. 
14. Tarasevich, B. N. IK spektry osnovnyh klassov organicheskih soedinenij. Spravochnye materialy [Text] / B. N. Tarasevich. - Moscow: MGU, 2012. -55 p.

15. Silverstein, R. M. Spectrometric identification of organic compounds. 7 edition [Text] / R. M. Silverstein, F. X. Webster, D. J. Kiemle. - John Wiley \& Sons Ltd, USA, 2005. -267 p.

\section{References}

1. Zelenin, K. N. (1998). Gidrazin. Sorosovskij obrazovatel'nyj zhurnal, 5, 59-65.

2. Mashkovskij, M. D. (2012). Lekarstvennye sredstva. 16th edition. Moscow: Novaja volna, 1216.

3. Sweetman, S. C. (Ed.) (2009). Martindale: The Complete Drug Reference. Thirty-sixth edition. Pharmaceutical Press, 3694.

4. Rollas, S., Küçükgüzel, S. G. (2007). Biological Activities of Hydrazone Derivatives. Molecules, 12 (8), 1910 1939. doi: 10.3390/12081910

5. Narang, R., Narasimhan, B., Sharma, S. (2012). A Review on Biological Activities and Chemical Synthesis of Hydrazide Derivatives. Current Medicinal Chemistry, 19 (4), 569612. doi: 10.2174/092986712798918789

6. Mosselhi, M. A. N., Tawfik, N. M., Shawali, A. S. (2003). New [ e ]-Fused Caffeines: A Simple Synthesis of 3Substituted [1,2,4]Triazolo[4,3- e ]purines. Monatshefte Fur Chemie/Chemical Monthly, 134 (4), 565-571. doi: 10.1007/ s00706-002-0514-7

7. Petch, D., Anderson, R. J., Cunningham, A., George, S. E., Hibbs, D. E., Liu, R. et. al. (2012). Design and synthesis of EGFR dimerization inhibitors and evaluation of their potential in the treatment of psoriasis. Bioorganic \&
Medicinal Chemistry, 20 (19), 5901-5914. doi: 10.1016/ j.bmc.2012.07.048

8. Ashour, F. A., Rida, S. M., El-Hawash, S. A. M., ElSemary, M. M., Badr, M. H. (2011). Synthesis, anticancer, antiHIV-1, and antimicrobial activity of some tricyclic triazino and triazolo[4,3-e]purine derivatives. Medicinal Chemistry Research, 21 (7), 1107-1119. doi: 10.1007/s00044-011-9612-6

9. Korobko, D. B., Berezovs'kyj, O. V., Palagnjuk, M. M., Pylypiv, Je. S. (2011). Syntez i fizyko-himichni vlastyvosti dejakyh 7-aralkil-(alkenil)-8-bromo-(tio-)teofiliniv. Liky - ljudyni. Harkiv, 263-269.

10. Abdel-Wahab, B. F., Abdel-Gawad, H., Mohamed, H. A., Dawood, K. M. (2010). Utility of 2,4-Dioxoesters in the Synthesis of New Heterocycles. HETEROCYCLES, 81 (1), 1-55. doi: 10.3987/rev-09-659

11. Gumennoj, V. P. (1984). Kondensacija 1,3-dikarbonil'nyh soedinenij s 8-gidrazinoksantinami. Chem. Abstrac., 101 (13), 110869.

12. Povstjanoj, M. V., Kruglenko, V. P., Kljuev, N. A. et. al. (1992). Kondensirovannye imidazo-1,2,4-aziny. XXVI. O reakcii 8-gidrazinoksantina i 8-(1-metilgidrazino)teofillina $\mathrm{s}$ acetil- i benzoilacetonom. Zhurnal org. himii, 28 (4), 849-856.

13. Voskobojnik, O. Ju. (2008). Syntez, peretvorennja, fizyko-himichni ta biologichni vlastyvosti [\{2-R-(3H)-[hinazolin-4-iliden \} gidrazono]karbonovyh kyslot. L'viv. nac. med. un-t imeni Danyla Galyc'kogo, 26.

14. Tarasevich, B. N. (2012). IK spektry osnovnyh klassov organicheskih soedinenij. Spravochnye materialy. Moscow: MGU, 55

15. Silverstein, R. M., Webster, F. X., Kiemle, D. J. (2005). Spectrometric identification of organic compounds. 7 edition. John Wiley \& Sons Ltd, USA, 267.

Рекомендовано до публікаиії д-р фарм. наук, професор Коваленко С. I. Дата надходження рукопису 09.02.2016

Korobko Dmytro, Candidate of Pharmaceutical Sciences, Associate Professor, Department of Pharmaceutical Chemistry. Dean of Pharmaceutical Faculty, I. Horbachevsky Ternopil State Medical University, 36 Ruska str., Ternopil, Ukraine, 46001

E-mail: kodibo.kdb@mail.ru 